\title{
O CONTROLE SOCIAL E A DEMOCRATIZAÇÃo dA INFORMAÇÃO: UM PROCESSO EM CONSTRUÇÃO
}

\author{
Marluce Maria Araújo Assis ${ }^{1}$ \\ Tereza Cristina Scatena Villa ${ }^{2}$
}

Assis MMA, Villa TCS. O controle social e a democratização da informação: um processo em construção. Rev Latino-am Enfermagem 2003 maio-junho; 11(3):376-82.

O presente trabalho tem como objetivo refletir criticamente sobre o controle social como uma conquista jurídicoinstitucional e as suas condições de legitimidade, apontando estratégias para a democratização da informação no sistema local de saúde. O texto foi desenvolvido em três eixos temáticos: o $1^{\circ}$ discute a participação social como conquista jurídicoinstitucional; o $2^{\circ}$ analisa as condições essenciais para a legitimidade e $03^{\circ}$ aponta a informação como elemento fundamental para a gestão e o controle social, como um processo inacabado, em construção.

DESCRITORES: sistema de saúde, participação comunitária, gerenciamento de informação

\section{SOCIAL CONTROL AND INFORMATION DEMOCRATIZATION: A PROCESS UNDER CONSTRUCTION}

This work aims at a critical reflection on social control as a legal-institutional achievement and its legitimacy conditions, pointing out strategies for information democratization in the local health system. The text was developed according to three thematic units: the first discusses social participation as a legal-institutional achievement; the second analyzes the essential conditions for legitimacy and the third presents information as a fundamental element for management and social control as an unfinished process that is still under construction.

DESCRIPTORS: health system, community, information management

\section{EL CONTROL SOCIAL Y LA DEMOCRATIZACIÓN DE LA INFORMACIÓN: UN PROCESO EN CONSTRUCCIÓN}

El presente trabajo tiene como objetivo reflexionar críticamente sobre el control social como una conquista jurídicoinstitucional y sus condiciones de legitimidad, describiendo estrategias para la democratización de la información en el sistema local de salud. El texto fue desarrollado en tres ejes temáticos: el primero discute la participación social como conquista jurídico-institucional, el segundo analiza las condiciones esenciales para la legitimidad y el tercero apunta la información como elemento fundamental para la gestión y el control social, como un proceso en construcción.

DESCRIPTORES: sistema de salud, participación comunitaria, gerencia de la información

\footnotetext{
${ }^{1}$ Professor Titular do Departamento de Saúde da Universidade Estadual de Feira de Santana-BA, Doutor em Enfermagem na Linha de Pesquisa Práticas, Saberes e Políticas de Saúde pela Escola de Enfermagem de Ribeirão Preto da Universidade de São Paulo, Coordenadora do Núcleo de Pesquisa Integrada em Saúde Coletiva (NUPISC) da Universidade Estadual de Feira de Santana, e-mail: aassis@uefs.br; ${ }^{2}$ Professor Livre Docente, Vice-Coordenadora do Grupo de Pesquisa "Estudos Operacionais em Tuberculose" - CNPq 2002, e-mail: tite@eerp.usp.br. Escola de Enfermagem de Ribeirão Preto da Universidade de São Paulo, Centro Colaborador da OMS para o desenvolvimento da pesquisa em enfermagem
} 
INTRODUÇÃO

A Constituição de $1988^{(1)}$, além de colocar como pauta de discussão da sociedade brasileira o direito à saúde, avança na compreensão desse conceito, passando a concebê-la enquanto resultante das condições de vida, assim como das formas de organização da produção. E, em sendo os serviços de saúde de relevância pública, cabe ao Estado a garantia das condições necessárias ao atendimento à saúde da população, ou seja, a saúde é um bem prioritário e uma condição para que o cidadão possa ter uma vida digna, produtiva e com qualidade.

Nesse sentido, o controle/participação social é entendido como um espaço de representação da sociedade, onde se articulam diferentes sujeitos, com suas diversas representações: movimentos populares, entidades de classe, sindicatos, governo, entidades jurídicas, prestadores de serviço, entre outros, e uma população com suas necessidades e interesses que envolvem 0 indivíduo, família e grupos da comunidade.

O controle social consiste, portanto, em canais institucionais de participação na gestão governamental, com a presença de novos sujeitos coletivos nos processos decisórios, não se confundindo com os movimentos sociais que permanecem autônomos em relação ao Estado.

A participação/controle social não pode ser entendida como uma extensão burocrática e executiva, mas como um processo contínuo de democratização no âmbito local, o que implica o estabelecimento de uma nova sociabilidade política e um novo espaço de cidadania, e, nesse sentido, o sujeito/cidadão deve ser o centro do processo de avaliação das ações nos serviços de saúde ${ }^{(2)}$.

Dito de outra maneira, a participação social é um campo em construção com "múltiplas possibilidades, de organização autônoma da sociedade civil, por meio das organizações independentes do Estado, tais como as associações de moradores, conselhos de saúde, associação de docentes, grupos de mulheres, que poderão confluir ou não suas intervenções para uma atuação direta junto aos órgãos de controle previstos em Leip(3).

Um ponto importante a considerar são as formas de efetivação do controle social na organização do Sistema Único de Saúde (SUS), representado pelos conselhos e conferências de saúde, enquanto instâncias que protagonizam a participação da comunidade na gestão do sistema, como um processo de articulação entre atores sociais que têm olhares distintos sobre a realidade e que são portadores de interesses diversos, nem sempre convergentes e harmônicos, em que as disputas de poder estão presentes, de conflitos e de negociação, ainda que os processos de discussão busquem o consenso em defesa dos princípios e diretrizes de um sistema público equânime e resolutivo.

No entanto, vale lembrar que nem sempre os consensos são possíveis, mantendo-se os conflitos e, nesse sentido, cabe a interferência de outros setores da sociedade, como o Ministério Público, que pode ser um aliado na construção do SUS, reforçando a participação de outros segmentos sociais na negociação política, enquanto instrumento de ação dos Conselhos de Saúde.

Em síntese, o controle social consiste num campo de relações sociais, no qual os sujeitos participam por meio de distintas articulações, que são processadas no âmbito interno e externo dos espaços institucionais, na busca da identificação de necessidades dos trabalhadores de saúde e grupos da comunidade em co-responsabilidade com as instâncias governamentais - Ministério da Saúde, Secretarias Estaduais e Municipais de Saúde.

Além disso, a informação seria um elemento fundamental para a gestão e o controle social do SUS, por meio do desenvolvimento e implantação de uma rede de informações que abranja todos os níveis de complexidade do sistema de saúde e promova a articulação entre as instituições produtoras de informações, de modo a relacionar os dados de saúde com bases de dados demográficos, socioeconômicos e financeiros, permitindo, por exemplo, a gerência informatizada das contas e a disseminação de estatísticas sanitárias, epidemiológicas e de atividades de produção de serviços, desenvolvidas nas instituições de saúde das esferas nacional, estadual, municipal e local.

Diante do exposto, justifica-se a discussão de controle social, como um tema que está na ordem do dia, por meio da atuação da sociedade civil organizada na gestão das políticas públicas, no sentido de controlá-las para que atendam as demandas e os interesses da coletividade, e, nessa perspectiva, o controle social requer a luta pelo fortalecimento do setor público, com ênfase no âmbito municipal. Esse âmbito é entendido, enquanto espaço de confronto de interesses, de identificação de necessidades e de construção de alternativas, até mesmo porque é no espaço dos municípios, onde os problemas são identificados com mais clareza e rapidez, assim como a solução a ser buscada em parceria com os segmentos 
sociais: gestores, trabalhadores de saúde e usuários dos serviços, na busca da qualidade do atendimento.

\section{OBJETIVO}

Refletir sobre o controle social como uma conquista jurídico-institucional e as suas condições de legitimidade, apontando estratégias para a democratização da informação no sistema local de saúde.

\section{O CONTROLE SOCIAL COMO CONQUISTA JURIDÍCO-INSTITUCIONAL}

Com o advento da Lei $n^{\circ}$ 8.142, de 28 de dezembro de $1990^{(4)}$, reconhece-se a participação da comunidade na gestão do SUS, mediante a criação dos Conselhos de Saúde e das Conferências de Saúde.

De fato, a Lei no 8.142 explicita como deve ser desdobrado o controle social, como se pode ver o conteúdo do disposto no artigo $1^{\circ}$ da referida Lei.

Art. 10 "O Sistema Único de Saúde... contará, em cada esfera de governo, sem prejuízo das funções do Poder Legislativo, com as seguintes instâncias colegiadas:

I-A Conferência de Saúde e

II - o Conselho de Saúde

$\S 1^{\circ}$ A Conferência de Saúde reunir-se-á a cada quatro anos, com representação dos vários segmentos sociais, para avaliar a situação de saúde e propor as diretrizes para a formulação da política de saúde nos níveis correspondentes, convocada pelo Poder Executivo ou, extraordinariamente, por este ou pelo Conselho de Saúde.

$\S 2^{\circ}$ O Conselho de Saúde, em caráter permanente e deliberativo, órgão colegiado composto por representantes do governo, prestadores de serviço, profissionais de saúde e usuários, atua na formulação de estratégias e no controle da execução da política de saúde na instância correspondente, inclusive nos aspectos econômicos e financeiros, cujas decisões serão homologadas pelo chefe do poder legalmente constituído em cada esfera do governo.

§ 3으은 Conselho Nacional de Secretários de Saúde (CONASS) e o Conselho Nacional de Secretários Municipais de Saúde (CONASEMS) terão representação no Conselho Nacional de Saúde.

$\S 4^{\circ}$ A representação dos usuários nos Conselhos de Saúde e Conferências será paritária em relação ao conjunto dos demais segmentos.

$\S 55^{\circ}$ As Conferências de Saúde e os Conselhos de Saúde terão sua organização por meio de normas de funcionamento definidas em regimento próprio, aprovado pelo respectivo Conselho."

Uma década depois, o SUS encontra-se regulamentado, e uma série de processos participativos foram desencadeados em vários "cantos" deste Brasil, se tomarmos os avanços da descentralização via municipalização da saúde e se examinarmos a ampliação do número de conselhos municipais, atendendo aos dispositivos legais das Leis no 8080 e no 8142 de 1990 .

Os dados apresentados pelo jornal do Conselho Nacional de Saúde ${ }^{(5)}$, em julho de 2000, são representativos do incremento do Controle Social, tomando como base o número de conselheiros efetivos em todo território nacional. Têm-se, portanto, 798 conselheiros estaduais e 2.780 municipais, dos 27 Estados da Federação e 5.507 municípios brasileiros.

O município representa um espaço privilegiado da luta social e política, os Conselhos de Saúde são instâncias públicas de formação de opinião e vontade política, muito mais do que instrumentos do governo ou da sociedade.

Os Conselhos de Saúde são órgãos colegiados, de caráter permanente e deliberativo. Definem o que deve ser feito, fiscalizam e avaliam o que foi feito. No exame das demandas e proposições dos segmentos sociais, os Conselhos não devem manifestar o interesse individual, deste ou daquele grupo, devendo ser julgadas a pertinência, a viabilidade, as prioridades, entre outros, de tal forma que o interesse individual se apresente e se habilite como interesse público.

Os Conselhos de Saúde devem representar e defender os interesses da sociedade, cumprindo a função de indutores de responsabilidade governamental, entendida como a qualidade dos governos de estar à altura da confiança e das expectativas dos cidadãos ${ }^{(6)}$.

A Lei o 8.142/1990 explicita a composição dos Conselhos de Saúde. Garante a representação dos seguintes segmentos: governo, prestadores de serviços, profissionais de saúde e usuários. Define, também, a paridade da composição de usuários em relação às demais representações, ou seja, $50 \%$ do número total de conselheiros será de representantes dos usuários, e os outros $50 \%$ será de representantes dos demais segmentos. 
O guia de referência para Conselheiros Municipais $^{(7)}$ informa a recomendação das vagas nos Conselhos de Saúde, segundo critérios estabelecidos pelo Conselho Nacional de Saúde, sendo assim distribuídas: $25 \%$ para trabalhadores de saúde e $25 \%$ para prestadores de serviços públicos e privados. Os conselheiros devem ser indicados pelas entidades ou movimentos a que pertencem, mediante discussão ampliada entre os pares ou outras entidades e movimentos afins.

O trabalho dos Conselhos de Saúde não deve ser confundido com o papel executivo do gestor municipal e estadual. Cabe ao Conselho propor a construção da política, no acompanhamento das ações, na fiscalização e utilização dos recursos e ao gestor a execução da política de saúde.

Os Conselhos de Saúde são instâncias internas à estrutura do SUS, e suas deliberações são, em geral, resultados de negociações com os órgãos executores (Ministério da Saúde, Secretaria Estadual e Municipal de Saúde), que devem ter como eixos orientadores à representatividade de seus membros, a visibilidade de suas proposições, a transparência das relações e articulação com a sociedade para garantir o processo democrático e a qualidade das ações.

Orelatório da $10^{\text {a }}$ Conferência Nacional de Saúde ${ }^{(8)}$ define, com clareza, o caráter deliberativo e o poder fiscalizador dos Conselhos de Saúde sobre as ações e serviços de saúde, com ênfase na democratização da informação: "livre acesso dos conselheiros de saúde a todas as informações sobre produção de serviços, horário de trabalho (especialmente plantões), custos de atendimento, indicadores de saúde, elaboração e implementação de Planos de Saúde, metas, propostas, programas, projetos, informações relativas ao saneamento básico e ao meio ambiente, contrato e convênios com a rede privada e filantrópica, implantação de consórcios intermunicipais, orçamento para a saúde e extratos bancários e fluxo de caixa diário das contas do Fundo de Saúde, auditorias e fiscalizações ou qualquer outra informação solicitada pelos conselheiros de saúde. A sonegação de informações deve implicar crime de responsabilidade".

Diante dessas proposições, são necessários esforços para possibilitar um sistema de gestão com coresponsabilidade, envolvendo gestores, profissionais de saúde e usuários, no qual todos devem assumir uma postura de compromisso com a "coisa pública", no sentido de dar maior visibilidade à gestão dos serviços de saúde. $E$, nesse campo de ação institucional, insere-se o controle social, partindo do reconhecimento de que “... não há 'controle' possível sobre organizações 'opacas', verticais, que não explicitam suas metas e seus critérios de qualidade e nas quais não existe responsabilização pelos atos individuais e de equipe"(3).

O controle social aqui não é tratado como forma de o Estado manter a ordem e coesão social em torno de um projeto hegemônico, mas a participação efetiva da sociedade sobre as ações do Estado, representada em última instância pelas instituições que são responsáveis pela gestão da saúde, cabendo, portanto, uma participação efetiva do sujeito/cidadão na definição das políticas de saúde no cenário local, regional e nacional.

\section{O CONTROLE SOCIAL: AS CONDIÇÕES PARA SUA LEGITIMIDADE}

O sistema de saúde brasileiro tem avançado com relação à descentralização de poder do nível federal para o estadual e municipal, bem como a democratização das políticas públicas, reconhecendo o município como um espaço autônomo da federação, transferindo-se para esse espaço novas responsabilidades e recursos públicos capazes de fortalecer o controle social e a participação da sociedade civil nas decisões políticas.

Coerente com esses propósitos, adota-se uma postura que possibilite a legitimidade do controle social, considerando algumas condições essenciais, conforme o guia de referência para conselheiros municipais ${ }^{(7)}$ :

- AUTONOMIA - a garantia das condições de funcionamento dos Conselhos de Saúde, do ponto de vista administrativo, financeiro e técnico. Devem ser garantidos no orçamento das Secretarias Municipais e Estaduais de Saúde recursos para o desenvolvimento do trabalho, desde a garantia do espaço físico até pessoal de apoio e administrativo para a execução das atividades, devendo ser valorizados, também, estudos de problemas detectados pelos representantes e elaboração de instrumentos que facilitem as decisões dos conselheiros.

- ORGANICIDADE - o nível de organização dos Conselhos, que é caracterizado pela infra-estrutura básica para realização dos encontros entre os conselheiros, da 
periodicidade, freqüência e regularidade dos participantes do Conselho às reuniões, entre outros. Os Conselhos devem ter instrumento administrativo próprio, como o Regimento Interno, que regulamente o seu funcionamento para orientar os processos de discussão, regularidade das reuniões, votação e outros encaminhamentos próprios das atividades dos conselheiros.

- PERMEABILIDADE - o estabelecimento de canais de recepção das demandas sociais, na identificação das demandas locais e das necessidades de saúde emergentes que deverão ser estabelecidas nas pautas de discussões dos Conselhos para serem negociadas a operacionalidade por parte do órgão executor - a Secretaria de Saúde.

- VISIBILIDADE - é um dos pontos centrais, sendo caracterizada pela transparência das ações dos Conselhos, na criação de canais de comunicação com a sociedade. Seria colocar o usuário no centro do processo, numa relação de co-responsabilidade (governo trabalhadores de saúde - prestadores de serviços e usuários). Daí a importância dos meios de comunicação e da criação de instrumentos que possibilitem a democratização da informação como boletins informativos, jornais, cartilhas e outros tipos de publicações, garantindo maior visibilidade e transparência.

Nessa perspectiva, os Conselhos de Saúde deveriam ser o exemplo vivo dos interesses da sociedade, dotados de legitimidade pública. Portanto, é preciso criar mecanismos para que a Lei deixe de ser apenas um instrumento legal e passe a concretizar um espaço democrático, com valorização da dimensão política com mais visibilidade, envolvendo, de forma mais direta e orgânica, a disseminação da informação, dando transparência ao processo governamental.

- ARTICULAÇÃO - a capacidade dos Conselhos de Saúde estabelecerem relações uns com os outros, no campo da saúde e em outras áreas sociais. Na saúde, a articulação deve envolver todas as representações: Conselho Local (das unidades de saúde), Conselhos Municipal, Estadual e Nacional, Comissões Intergestores Bipartide e Tripartide, Conselhos de Secretários Municipais e Estaduais de Saúde, bem como as instituições da área da saúde. Além disso, é importante a articulação com outros setores da sociedade como, por exemplo, a Câmara de Vereadores e Assembléias Legislativas, movimentos populares e o Ministério Público. Estas relações fortalecem as ações dos Conselhos e podem formar um bloco de forças sociais em defesa dos interesses da sociedade.

O Ministério Público é um órgão da Administração Pública responsável pela defesa da ordem jurídica, do regime democrático e dos interesses sociais e individuais. E por ter competência jurídica, é um órgão importante na construção e legitimidade do SUS, sendo, portanto, indispensável como interlocutor do controle social.

O Conselho é um espaço de articulação política, onde os atores principais negociam as situações em busca de soluções para os problemas de saúde da população dos bairros, distritos e municípios.

Outra forma de controle social - as Conferências de Saúde -, estas são fóruns de representação ampla e democrática dos diversos segmentos sociais: usuários do SUS, profissionais de saúde, gestores, prestadores dos serviços de saúde, parlamentares e outros, com a função de avaliar e propor diretrizes à formulação de políticas de saúde nos três níveis de governo (nacional, estadual e municipal).

Assim, foram realizadas três Conferências Nacionais de Saúde no período de 1986 a $1996^{(9)}$. A $8^{\text {a }}$ Conferência, realizada em 1986, teve um papel fundamental na construção do SUS. O seu relatório final serviu de base para aprovação do Capítulo Saúde, na Constituição de 1988, nos Artigos 196, 197, 198, 199, 200 e $227^{(4)}$.

Em 1992, foi realizada a 9 $^{\text {Conferência }}{ }^{(10)}$, fortalecendo o cumprimento da Lei que rege o SUS, e a $10^{\text {a }}$ Conferência, em $1996^{(8)}$, reafirmou o Sistema Único de Saúde sob a lógica da solidariedade, pautado em uma universalização não excludente, em uma descentralização financeira, de poder e de autonomia relativa aos municípios para melhoria da eficiência e eficácia dos serviços ao nível local; eqüidade - que confira a igualdade de insumos e de acesso por necessidades iguais, enquanto valor normativo de estruturação do sistema de saúde -; participação e controle social, compreendidos esses espaços enquanto instâncias problematizadoras, de exercício da democracia e da atualização de identidade das lutas de saúde no país.

A 11aㅡ Conferência Nacional de Saúde ${ }^{(11)}$, realizada em dezembro de 2000, teve como finalidade reforçar as diretrizes das Conferências anteriores. $O$ tema central do encontro intitulou-se: Efetivando o SUS: Acesso, Qualidade e Humanização da Atenção à Saúde com Controle Social. Os participantes indicam os avanços e colocam os desafios que devem ser enfrentados para que se alcance o objetivo de concretizar o SUS. 
O CONTROLE SOCIAL E A DEMOCRATIZAÇÃO DA INFORMAÇÃO: UM PROCESSO EM CONSTRUÇÃO

O controle social tem papel fundamental enquanto princípio orientador do SUS que possibilite a participação da sociedade na formulação, execução e fiscalização das políticas de saúde.

Portanto, o controle social e a democratização da informação se reforçam mutuamente. Teria que haver uma confluência entre o interesse do usuário em acompanhar e exigir uma boa qualidade do serviço prestado pelo SUS, com o interesse do serviço em informar e ser informado sobre suas falhas e inadequações, no esforço de aprimorar, cada vez mais, suas ações e possibilitar visibilidade e transparência no exercício da prática cotidiana.

Nesse processo de democratização da informação, seria necessário tomar como eixo central as necessidades de saúde do sistema local. Entendendo por necessidades de saúde as demandas individuais, de grupos da comunidade e famílias que buscam ativamente por intervenção nos serviços de saúde, a partir de carecimentos reconhecidos pelos indivíduos em seu atual estado sociovital. Pode ser uma alteração física, orgânica ou psíquica, "... que os impedem de seguir vivendo em sua rotina de vida, ou um sofrimento ainda não identificado fisicamente; ou até mesmo uma situação que reconhece como 'uma falta', algo de que carece, como por exemplo, uma informação"(12).

Questões referentes aos sistemas de informação no Brasil, especialmente no âmbito municipal, constituem, ainda hoje, um dos principais problemas para a organização de um sistema de saúde na perspectiva da Vigilância à Saúde presente nas diretrizes do SUS. Apesar dos recentes avanços advindos da descentralização das ações de saúde, os gestores do SUS têm como desafio fazer com que as informações sobre a saúde e sobre o SUS cheguem a todos os setores da sociedade, principalmente os usuários, por meio de meios acessíveis e diretos, como, por exemplo ${ }^{(7)}$ : rádio, televisão, jornal, internet, e outros meios de fácil acesso, permitindo maior agilidade na informação e orientação da população; mensagens em linguagem popular, em folder e materiais utilizados nas escolas, em revistas em quadrinhos e outras de grande circulação e/ou de penetração em públicos específicos; plenárias de discussão e divulgação do SUS nos estados e municípios; programas educativos veiculados pela mídia, esclarecendo o que é o SUS, o que faz e como faz.

Algumas estratégias podem ser utilizadas com a finalidade de tornar públicas as informações sobre atendimento em cada uma das unidades prestadoras de serviços do SUS, tais como: fixação, em local visível e de fácil acesso aos usuários, em todas as unidades e serviços de saúde do SUS, de informações sobre as ações oferecidas, horários de funcionamento, quadro de trabalhadores e seus horários e funções; publicações contendo informações sobre a capacidade instalada e as formas de acesso aos serviços da rede própria, conveniada e contratada do SUS ${ }^{(7)}$.

Por outro lado, pode-se afirmar que existem dificuldades na construção de um sistema de informações, considerando que os dados registrados rotineiramente pelos serviços de saúde, muitos deles não são utilizados. $\mathrm{Na}$ ausência de análise e incorporação dessas informações nos processos decisórios, outros parâmetros são utilizados na orientação das políticas - nem sempre esses parâmetros voltam-se para o atendimento das necessidades da população. Ao contrário, na maioria das vezes, esses parâmetros estão articulados apenas à lógica mercantilista aplicada à área de saúde (entendida, aqui, como a produção de serviços que tem, como finalidade última, o lucro). Portanto, a construção de sistemas complexos de alimentação de dados, análise e sistematização sintonizada com aspectos financeiros e contábeis direcionam-se, predominantemente, à contabilização de procedimentos e ações para fins de remuneração dos serviços e dizem respeito à aferição de custos da utilização da rede ambulatorial e hospitalar, operando, exclusivamente, como instrumento administrativo. Essas informações registradas nada têm em comum com o novo modelo assistencial que se deseja implementar, no qual as informações registradas seriam utilizadas para análise da situação de saúde, para a definição de prioridades e reorientação das práticas.

Em linhas gerais, o sistema de informação a ser implementado deve proporcionar às equipes dos distritos e das unidades de saúde informações epidemiológicas e socioeconômicas desagregadas e distribuídas no espaço territorial local. Uma das estratégias seria a territorialização das informações para estimular um processo de mudança nas atitudes das equipes dos serviços, no entendimento de que o compromisso dessas últimas é com a saúde da 
população que reside numa determinada área geográfica, coberta pelo serviço, e não apenas com a produção de procedimentos preventivos e assistenciais dentro do espaço físico do estabelecimento de saúde.

Além disso, há necessidade de detectar desigualdades na oferta de serviços com o objetivo de subsidiar um enfoque mais eqüitativo - coerente com as necessidades - para os processos de planejamento, programação e alocação de recursos, apresentando à população, de maneira clara e facilmente inteligível, os dados referentes aos principais problemas de saúde, assim como os compromissos assumidos pelas equipes dos serviços - com o propósito de garantir uma participação da sociedade civil nas decisões e de facilitar o controle social por meio de um incremento da transparência institucional.

Algumas iniciativas têm sido tomadas para o fortalecimento de uma rede que possibilite disponibilização de informações voltadas ao conhecimento e compreensão da realidade sanitária brasileira, bem como suas perspectivas no contexto local. As salas de situação retratam essas iniciativas importantes e consiste em instrumento de grande relevância no exercício do controle social, tendo em vista a utilização de instrumentos de ação por meio de dados demográficos, geográficos, epidemiológicos, socioeconômicos, financeiros e de

\section{REFERÊNCIAS BIBLIOGRÁFICAS}

1. Constituição da República Federativa do Brasil 1988. São Paulo: Ed. Revista dos Tribunais; 1989.

2. Assis MMA, Kantorski L, Tavares JL. Participação social: um espaço em construção para a conquista da cidadania. Rev Bras Enfermagem 1995 outubro-dezembro; 48(4):32940.

3. Cecílio LCO. Pensando mecanismos que facilitem o controle social como estratégia para a melhoria dos serviços públicos de saúde. Rev Saúde em Debate 1999 setembrodezembro; 23(53):30-6.

4. Carvalho GI, Santos L. Sistema Único de Saúde: comentários à Lei Orgânica da Saúde (Lei 8.080/90 e Lei 8.142/90). 2.ed. São Paulo (SP): HUCITEC; 1995.

5. Ministério da Saúde.(BR) Controle social. J Conselho Nac Saúde julho 2000; 8(3).

6. Carvalho Al. Conselhos de saúde, responsabilidade pública e cidadania: a reforma sanitária como reforma do estado. In: Fleury S, organizadora. Saúde e democracia a luta do CEBES. São Paulo (SP): Lemos Editorial; 1997. p. 93-111. 7. Ministério da Saúde (BR). O SUS e o controle social: guia de referência para conselheiros municipais. Brasília; 1998. produção de serviços que deverão ser expostos para os Conselhos de Unidades e Municipais de Saúde, trabalhadores de saúde e qualquer cidadão interessado em constituir parte das informações; bem como na construção de home-pages municipais.

Portanto, se o desafio é instituir um sistema público de saúde democrático, onde as prioridades sejam definidas em gestões colegiadas e participativas, com mecanismos de controle social, é urgente a definição de uma política de informação em saúde que garanta a comunicação e divulgação de dados consistentes e transparentes em articulação com instituições de saúde e áreas afins que possam contribuir na identificação, processamento e análise crítica de dados e indicadores de saúde, permitindo o aprofundamento de aspectos ainda pouco explorados ou não identificados, como de relevância para a compreensão do quadro sanitário brasileiro.

As participações sociais ativas, informadas e democráticas, são processos inacabados. A cada dia, questionamos, revisamos e ampliamos nossas concepções e ações. Foi com essa certeza (ainda que provisória) que escrevemos este artigo, com a esperança e a crença de que a saúde é um valor humano inquestionável, um direito básico de cidadania, que é construído por sujeitos individuais e coletivos, no cotidiano institucional e social.

8. Ministério da Saúde (BR). Relatório final da $10^{\text {a }}$ Conferência Nacional de Saúde. Brasília; 1996.

9. Correia MVC. Que controle social? Os conselhos de saúde como instrumento. Rio de Janeiro (RJ): FIOCRUZ; 2000.

10. Ministério da Saúde (BR). Relatório final da IX Conferência Nacional de Saúde. Brasília; 1993.

11. Ministério da Saúde (BR). Relatório final da 11a Conferência Nacional de Saúde. Efetivando o SUS: acesso, qualidade e humanização da atenção à saúde com controle social. Brasília; 2001.

12. Schraiber LB, Mendes-Gonçalves RB. Necessidades de saúde e atenção primária. In: Schraiber LB, Nemes MIB, Mendes-Gonçalves RB, organizadores. Saúde do adulto programas e ações na unidade básica. São Paulo (SP): HUCITEC; 1996. p.29-47. 